\title{
SISTEMA DE COMUNICACIÓN DE RESPALDO MEDIANTE TECNOLOGÍA LORA CON HARDWARE Y SOFTWARE ABIERTO PARA APLICACIONES DE ROBÓTICA DE EMERGENCIAS
}

\author{
R.F. Manrique Balmaceda, R. Vázquez Martín, J. Bravo Arrabal, J.J. Fernández Lozano, A. García Cerezo \\ Departamento de Ingeniería de Sistemas y Automática. Universidad de Málaga. \\ C/ Dr. Ortiz Ramos, s/n, 29071, Málaga. \\ jfl@uma.es
}

\begin{abstract}
Resumen
Los sistemas de comunicación entre nodos sensores y los equipos de búsqueda y rescate presentan posibles vulnerabilidades ante emergencias o catástrofes, como el fallo de la infraestructura del sistema o la saturación de la red. Por ello, es necesario evaluar alternativas para garantizar la disponibilidad del sistema y los datos necesarios para la estrategia del equipo. Los requerimientos de una red de largo alcance y bajo consumo apuntan a evaluar las distintas tecnologías que engloban las redes LPWAN (Low Power, Wide Area Network). Entre ellas, destaca el protocolo LoRaWAN (Long Range, Wide Area Network) por ofrecer una flexibilidad operativa que no tienen los demás. De lado del hardware, se realizó una selección de componentes abiertos para los nodos sensores y el gateway. Para conseguir una red ad-hoc se ha usado el proyecto ChirpStack como servidor de red y de aplicación. Finalmente, se ha integrado el software de aplicación QGIS con la base de datos PostgreSQL que almacena los datos en la misma tarjeta host del gateway. El sistema fue probado en las XV Jornadas Internacionales de la Universidad de Málaga sobre Seguridad, Emergencias y Catástrofes. El código y la documentación del sistema presentado en este artículo está disponible en https://github.com/jjflozano/BackUpCommLoRa.
\end{abstract}

Palabras clave: redes de sensores inalámbricas, robots móviles, LoRaWAN, SAR, sistema de comunicación de respaldo, red ad-hoc, Chirpstack

\section{INTRODUCCIÓN}

El uso de robots como parte de los equipos de búsqueda y rescate (Search and Rescue, SAR) en emergencias y catástrofes es relativamente reciente. El primer caso importante fue su participación en el atentado del World Trade Center (WTC) de Nueva York (2001), en el cual sirvieron principalmente como transmisores de imágenes para reconocimiento de víctimas. Desde ahí, la tecnología ha seguido progresando y los robots han incorporado nuevas funciones que han hecho que la labor de rescate sea cada vez más eficaz y eficiente [1].
La Universidad de Málaga (UMA) cuenta con un área de investigación en robótica de rescate, haciendo uso de robots terrestres y aéreos como parte de los equipos de emergencias en eventos catastróficos. Las actividades de primer contacto del equipo de respuesta con la zona de emergencia se denominan "primera intervención" e incluyen la recopilación de información relevante para el planteamiento de las estrategias de rescate. Una de las líneas de investigación son los sistemas de comunicación entre robots, nodos sensores y equipos SAR [2].

Otro elemento relevante de los equipos de rescate son los perros. Su capacidad para localizar víctimas, y para llegar a áreas de difícil acceso para humanos los hacen elementos muy valiosos de las operaciones de búsqueda y rescate. Asimismo, pueden portar, como los robots, sensores que complementen la información sobre la zona de operaciones. Por ejemplo, en 2018 se integraron nodos sensores de gases $\left(\mathrm{O}_{2}, \mathrm{O}_{3}, \mathrm{CO}_{2}, \mathrm{CO}\right.$, $\mathrm{NH}_{3}$ y VOC), humedad, presión atmosférica y temperatura, así como un GPS, en un perro de rescate [3]. El estándar de comunicación utilizado fue ZigBee con un alcance máximo de $700 \mathrm{~m}$ (con línea de vista directa). Los nodos se comunican con una puerta de enlace, y desde ahí se enviaba la información integrada a un servidor mediante comunicación celular $(3 G)$.

Otro proyecto más reciente $(2020)$ ha sido la implementación de un sistema de comunicación híbrido usando gateways de tecnología LoRa estáticos y móviles. En este trabajo se evaluaron distintas configuraciones del protocolo LoRaWAN para los nodos y gateways instalados por la ciudad de Málaga (incluyendo los componentes móviles instalados en un automóvil eléctrico) [4].

Hoy en día la oferta de tecnologías de comunicaciones se ha incrementado con protocolos que tienen como característica principal un largo alcance directo como las redes LPWAN y las redes móviles 5G que logran una gran capacidad de transmisión de datos, en comparación con sus predecesoras.

Este trabajo presenta la implementación de un sistema de comunicación, desarrollado con el objetivo de servir como red de respaldo a los sistemas de 
comunicación principales, particularmente en cuanto a la transmisión de datos de sensores desde el campo de operaciones hasta los centros de coordinación, debido a que en las misiones de búsqueda y rescate con frecuencia la infraestructura está afectada.

Además, el sistema está basado en elementos de hardware y software abierto por su flexibilidad y modularidad, así como por el reducido coste, que facilita replicar los elementos básicos de la red para poder contar con un elevado número de nodos.

\section{SISTEMA DE COMUNICACIONES}

\subsection{REQUERIMIENTOS DE DISEÑO}

En relación a la aplicación, la comunicación entre los nodos sensores y el equipo SAR requiere las siguientes características [5]:

$\checkmark$ Redes Ad-hoc: al ser un sistema de respaldo, la comunicación debe ser independiente de sistemas existentes. Este requisito es específico de este trabajo.

$\checkmark \quad$ Largo alcance: por la posible extensión del área impactada (sobre todo en desastres naturales, como terremotos).

$\checkmark \quad$ Buena capacidad de penetración: los despojos y restos típicos de catástrofes pueden atenuar considerablemente la señal.

$\checkmark$ Bajo consumo energético: una red de sensores móviles requiere de un uso eficiente de la energía, puesto que de este recurso dependerá el tiempo de autonomía de las unidades.

\subsection{SELECCIÓN DEL PROTOCOLO DE COMUNICACIÓN}

Para seleccionar el protocolo en el que se basa el sistema propuesto se ha realizado un análisis de las tecnologías LPWAN más relevantes. Entre ellas destaca: LoRa, Sigfox, Ingenu RPMA, Weightless, NB-IoT, Dash7, EC-GSM-IoT y LTE-M.

A igualdad de potencia de transmisión, la tecnología LoRa (Long Range) e Ingenu RPMA (Random Phase Multiple Access) tienen muy buen alcance porque usan técnicas de modulación de espectro ensanchado. LoRa utiliza la técnica Chirp Spread Spectrum (CSS) mientras que Ingenu usa la técnica Direct Sequence Spread Spectrum (DSSS). La ventaja de este tipo de tecnología es su baja afectación a interferencias o ruido, así el dispositivo receptor puede captar la señal incluso con niveles muy bajos de sensibilidad ( - 148 $\mathrm{dB})$.

Los estándares Weightless y NB-IoT (Narrow Band, Internet of Things) usan canales con ancho de banda estrecho (Narrow Band, NB) y Sigfox, ancho de banda ultra-estrecho (Ultra Narrow Band, UNB). Esta característica también permite al receptor recibir mensajes con muy baja potencia de transmisión (buena sensibilidad), pues el nivel de ruido en un canal muy estrecho es más fácil de filtrar y el manejo del ancho de banda total se hace más eficiente (más canales por ancho de banda) [6].

La transmisión con una frecuencia portadora en la banda sub-GHz es favorece la penetración de la señal a través de obstáculos físicos. Este rango de frecuencias pertenece a las comunicaciones móviles (700, 800 y $900 \mathrm{MHz}$ ), a la banda ISM (Industrial, Scientific and Medical) de $433 \mathrm{MHz}$ y a la banda de baja frecuencia de $868 \mathrm{MHz}$ [7]. En comparación con la banda ISM de $2.4 \mathrm{GHz}$ (WiFi, ZigBee, Bluetooth), las frecuencias sub-GHz tienen menos interferencia porque las tecnologías móviles asignan canales específicos de su ancho de banda contratado, y también porque las bandas ISM y de baja potencia tienen restricciones de uso que limitan la contaminación del espectro (baja velocidad de transmisión y potencia de emisión) [8].

El estándar Ingenu RPMA transmite a una frecuencia de $2.4 \mathrm{GHz}$ por las menores limitaciones en el uso del espectro (duty cycle) en comparación con la franja ISM y de baja potencia. La contrapartida es el mayor nivel de interferencia en el espectro. Aunque pueda mantener enlaces de hasta $15 \mathrm{~km}$ en un área urbana [4], [8], la baja penetrabilidad por la frecuencia portadora afectaría la potencia de transmisión disponible del enlace en esta aplicación.

En relación al protocolo Dash7, su punto débil principal es su bajo alcance frente a otros protocolos. El alcance entre nodo y gateway es de $1 \mathrm{~km}$, y puede extenderse hasta $2 \mathrm{~km}$ con el uso de un subcontrolador [9].

Como se ha mencionado, la red de comunicación es ad-hoc, por lo que no se utilizará estándares que requieran de compañías prestadoras de servicio, tal como las compañías de comunicaciones móviles (ECGSM-IoT, LTE-M, NB-IoT) o compañías que operen en las bandas ISM y de baja potencia pero que requieran un pago por su servicio (Sigfox e Ingenu RPMA). Solo las tecnologías Weightless, LoRa y Dash7 cumplen con la característica de red ad-hoc.

En relación a la modularidad, la tecnología LoRa lleva más de cinco años en desarrollo y cuenta con módulos compatibles que pueden ser conectados directamente con la tarjeta principal tarjeta host del nodo y del gateway. Además, el estándar LoRaWAN es de licencia abierta, por lo que no requiere pago alguno por su uso y tiene un soporte técnico que se encarga de su actualización y mejora [10]. 
Por las razones analizadas, el protocolo LoRaWAN es el elegido para este trabajo.

\subsection{PROTOCOLO DE COMUNICACIÓN LORAWAN}

El protocolo de comunicación LoRaWAN determina las reglas y la arquitectura de la red (estándares de acceso al medio) para su comunicación usando la tecnología LoRa, la cual determina los parámetros técnicos y metodologías requeridas por la capa física del sistema. La técnica de modulación de la tecnología LoRa es la clave de las particulares prestaciones técnicas del protocolo LoRaWAN: comunicación inalámbrica de largo alcance y bajo consumo de energía para una aplicación de baja velocidad de transmisión. se denomina Chirp Spread Spectrum (CSS).

La topología más amplia del protocolo LoRaWAN es Estrella de Estrellas (Figura 1: Topología del protocolo LoRaWAN). Varios nodos finales o nodos sensores envían la información a todos los gateways que se encuentren a su alcance: ésta es la primera estrella. La segunda está compuesta por todos los gateways conectados por internet a un mismo servidor. En este trabajo solo hace falta una estrella debido a la restricción de uso de internet (red ad-hoc). Por lo tanto, el alcance de la red estará supeditado al alcance de los transceptores LoRa.

Los gateways sirven de "túneles" por donde los nodos sensores pasan la información al servidor de red [11], pudiendo operar en local (LAN), o a través de Internet (WAN). En ambos casos, se hace uso de un servidor MQTT (Message Queue Telemetry Transport) que, para este trabajo, ha estado alojado en el propio host del gateway. La identificación y gestión de los nodos sensores, así como de los procesos de enlace, son realizados por los servidores de red y aplicación.

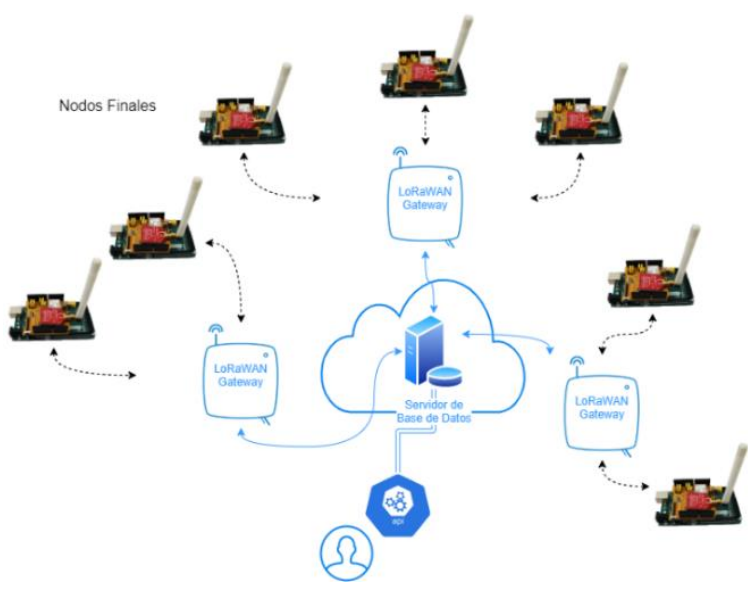

Figura 1: Topología del protocolo LoRaWAN
Cuando la información está disponible en el servidor, éste la enviará al servidor de aplicación y desde aquí el usuario podrá acceder a la información que necesite a través de la aplicación (una aplicación móvil, una interfaz web, un software, etc.).

El servidor de red autentica los mensajes recibidos y los retransmite al servidor de aplicación. Por ejemplo, si un nodo sensor envía un mensaje a dos gateways, el servidor de red se encarga de eliminar los mensajes duplicados.

Finalmente, el servidor de aplicación se encarga de recibir los mensajes del servidor de red para encriptar o desencriptar el mensaje generado en el nodo sensor.

\section{DISEÑO Y SELECCIÓN DE COMPONENTES}

\subsection{SELECCIÓN DEL HARDWARE}

El nodo sensor tiene características especiales que lo diferencian de los otros componentes. Pertenece a una unidad móvil, lo que condiciona que su diseño debe optimizar el consumo energético, el peso y volumen (sobre todo, al ir montado en unidades aéreas) y que las aplicaciones típicas suelen contar con más de un nodo sensor [12]. A medida que el número de nodos aumenta, el coste del equipo se vuelve más relevante. A continuación, se listan cuatro características usadas para la selección de las componentes del nodo sensor [13]:

$\checkmark \quad$ Utilización de microcontroladores de tecnología abierta.

$\checkmark$ Flexibilidad y modularidad de las tarjetas.

$\checkmark$ Bajo consumo energético.

$\checkmark \quad$ Bajo coste del hardware.

El nodo o dispositivo final está compuesto por los siguientes bloques (en la Figura 2: Diagrama de bloques del nodo sensor

se muestra un diagrama de bloques típico de un nodo sensor con una arquitectura modular) [13]:

\footnotetext{
$\checkmark \quad$ Bloque de procesamiento (host).

$\checkmark$ Bloque de sensores

$\checkmark$ Bloque de comunicaciones.

$\checkmark$ Bloque de alimentación.
}

El prototipo del nodo sensor está formado por una tarjeta principal (host), cuyo microcontrolador se comunica con las tarjetas secundarias o shields. La modularidad permite una conexión segura y una unidad compacta. 
La tarjeta host debe contar con múltiples interfaces de comunicación para la conexión con el módulo LoRa y con los módulos de sensores. Las interfaces más utilizadas son UART, SPI e I2C.

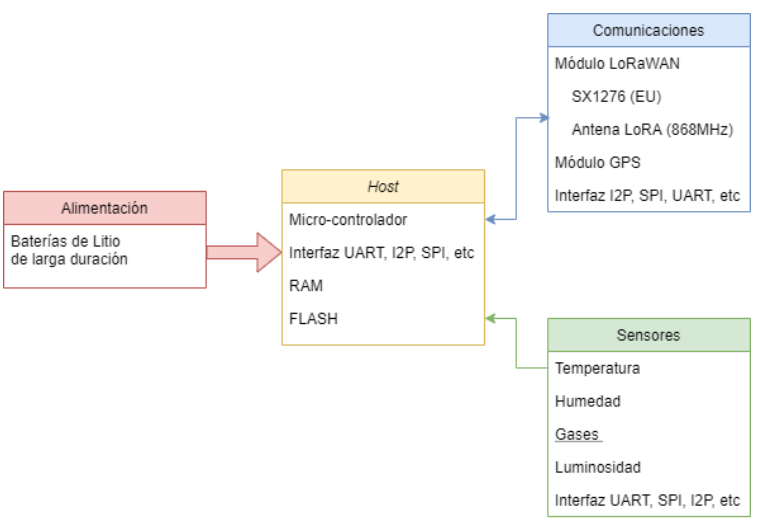

Figura 2: Diagrama de bloques del nodo sensor

El código utilizado para los nodos sensores es corto y sencillo, por lo que no hay inconvenientes con los requerimientos para la velocidad de procesamiento del microcontrolador, y cubre la mayoría de estos sin problema. Lo mismo sucede con los requisitos de hardware, pues para este tipo de aplicación no se requiere memoria RAM de gran capacidad.

La tarjeta host seleccionada es la Arduino UNO, que cuenta con una CPU AVR de tecnología CMOS (bajo consumo) y con arquitectura RISC (no requiere mayor complejidad para su aplicación). La tarjeta Arduino UNO es un módulo comercial y compatible con módulos de comunicación y de sensores, con lo que se garantiza la compacidad de la unidad. Otra alternativa es la tarjeta Arduino MEGA2560 (usada de hecho en varios experimentos), más grande en tamaño y con mejores prestaciones, pero que cumple con las mismas características de robustez y modularidad (existen módulos sensoriales LoRa que se conectan directamente), por la facilidad para su abastecimiento y flexibilidad de uso. Respecto a la evaluación económica, Arduino UNO es una de las tarjetas más baratas del mercado.

Dado que el criterio prevalente es la flexibilidad y modularidad, se ha elegido el módulo LoRa/GPS Shield v1.3 de Dragino para Arduino Uno, Leonardo y Mega2650. Junto con el módulo LoRa BEE v1.1 forman la unidad de comunicación LoRa y GPS. La tarjeta LoRa Bee tiene una arquitectura similar al módulo RFM95 de HopeRF. En la Figura 3 se muestra la tarjeta host y la tarjeta de comunicación acopladas.

Para la tarjeta host del gateway se eligió una Raspberry Pi 3B ya que requiere recursos informáticos superiores para la comunicación (niveles de Red y Transporte) difíciles de solventar con microcontroladores [13]. Un requerimiento más es la capacidad de almacenamiento de datos, teniendo en cuenta que la cantidad de mensajes de distintos nodos y por un periodo prolongado (días). También se ha considerado su disponibilidad comercial, modularidad con las tarjetas de comunicación LoRaWAN y coste.

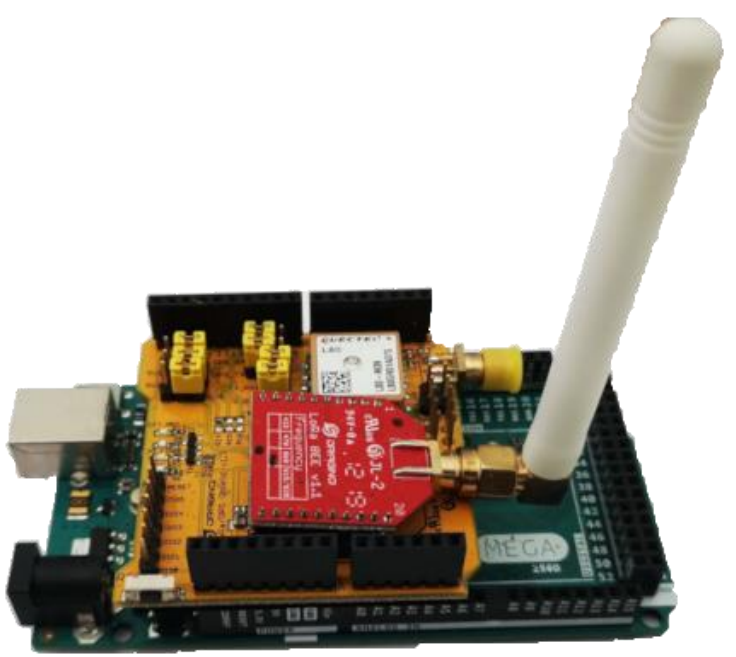

Figura 3: Prototipo del nodo sensor

El gateway está constituido por un host Raspberry Pi 3B que puede funcionar directamente conectado con el shield RAK2245 RPi HAT o con el módulo iC880A-SPI LoRa, en adelante iC880A, el cual se conecta mediante cables con la placa principal Raspberry Pi 3B. Para este trabajo se ha utilizado la tarjeta iC880A.

Para el bloque de sensores se ha empleado una tarjeta Proto Shield en la que se soldó el sensor LM35 y posteriormente se conectó en la parte superior de la tarjeta de comunicación LoRa.

\subsection{PROGRAMACIÓN}

El código del nodo sensor está escrito en $\mathrm{C}$ e incluye la librería Arduino-LMI que contiene las funciones asociadas al transceptor LoRa SX1276. Mediante el puerto serie y analógico recibe los datos del GPS y de los sensores respectivamente, y los transmite por el puerto ICSP hacia el módulo LoRa.

El código usado para el gateway está basado en el original de la compañía Semtech Corporation adaptado para la tarjeta iC880A (https://github.com/ttn-zh/ic880a-gateway.git). Estos ficheros están configurados para trabajar con el servidor TTN. Sin embargo, modificando la dirección IP del fichero de configuración también puede enlazarse con un servidor local.

Para crear una red Ad-Hoc se ha decidido disponer localmente del servidor de red y de aplicación. El 
servidor LoRa utilizado ha sido desarrollado por ChirpStack (https://www.chirpstack.io/), el cual es un servidor de red open-source con licencia MIT. El servidor ChirpStack está compuesto por los siguientes componentes:

$\checkmark \quad$ Puente (Bridge): servicio que convierte los paquetes UDP en el formato común JSON.

$\checkmark \quad$ Servidor LoRa: gestiona la recepción de los mensajes de subida (uplink) y la duplicación de paquetes provenientes del gateway, administra la capa MAC y programa los mensajes de bajada (downlink).

$\checkmark \quad$ Servidor de Aplicación LoRa: gestiona la solicitud de admisión (join request), encriptación de la información enviada por los clientes y ofrece la información solicitada por los clientes a través de una interfaz web.

Después de que un nodo sensor envíe un paquete de datos al gateway, este lo envía al servidor en formato UDP. El paquete ingresa por el puente, el cual publica la información en el bróker MQTT, el servidor de base de datos recibe la información del bróker MQTT y lo almacena. Cuando un usuario final solicita información a través de una API, el servidor de aplicación pide la información al servidor de base de datos y la retransmite al usuario final. En la Figura 4 se muestran las componentes del servidor ChirpStack y el flujo de información.

Finalmente, el software de aplicación usado es QGIS para la representación geoespacial de los datos medidos por el sensor y pgAdmin como gestor de la base de datos PostgreSQL (Figura 5). El servidor de aplicación de ChirpStack tiene una integración PostgreSQL lo que permite la conexión con el software QGIS y la visualización georreferenciada de la información de los nodos sensores. El software pgAdmin es un gestor de PostgreSQL para visualizar los datos de manera más amigable. El servidor
ChirpStack también cuenta con una interfaz web para visualizar los datos recibidos.

\section{PRUEBAS DE CAMPO}

El 18 de junio de 2021 se celebraron las XV Jornadas Internacionales de la Universidad de Málaga sobre Seguridad, Emergencias y Catástrofes. Dentro de las actividades se incluyeron las pruebas del sistema de comunicaciones LoRa con un nodo sensor.

El gateway iC880A se instaló en el Puesto de Control Avanzado (PCA, Figura 6), en un área cercana a la zona afectada. El nodo sensor se montó sobre el dron DJI Matrice 600 que realizó un barrido de un área determinada (Figura 7). El nodo envió la temperatura en un intervalo de 10 segundos y con spread factor igual a 7. Para una segunda prueba, el nodo sensor se montó en el robot terrestre Cuadriga II y el spread factor se cambió a 12 para un mayor alcance, a costa de un mayor consumo energético.

En la Figura 8 se muestran los puntos medidos por el nodo sensor, la interfaz gráfica es el software QGIS. Cada vez que la base de datos registra un nuevo dato, QGIS actualiza la capa vectorial que contiene cada uno de los puntos georreferenciados, su temperatura y también se añadió el dato de sensibilidad de la señal (RSSI).

La máxima sensibilidad registrada con un Spread Factor de 7 es de $-121 \mathrm{~dB}$, pudiendo alcanzar incluso más sensibilidad si es que el Spread Factor se incrementa. La razón por la que se inició con este valor fue para asegurar que se cumplieran los intervalos de tiempo establecidos en el código del nodo sensor (10 segundos). Se comprobó que cuando se varió a 12, el intervalo de tiempo creció considerablemente a aproximadamente 2 minutos, debido a la limitación de uso de los canales, impuesta por la banda ISM (1\% del duty cycle).

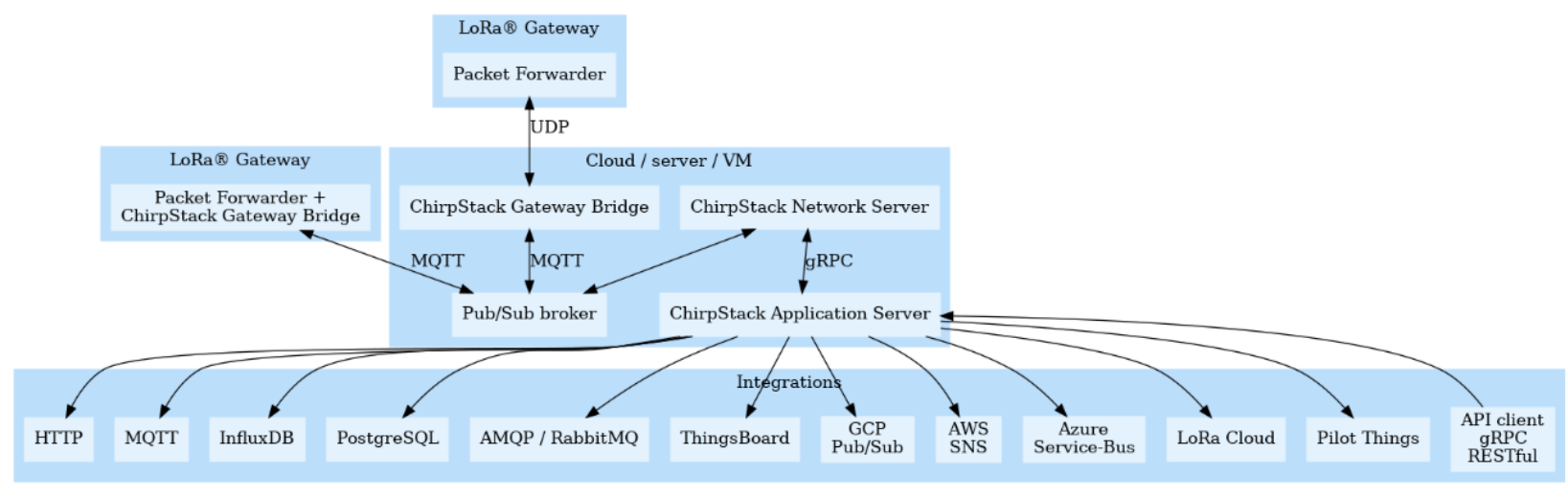

Figura 4: Estructura interna del Servidor ChirpStack Fuente: https://www.chirpstack.io/project/architecture/ 


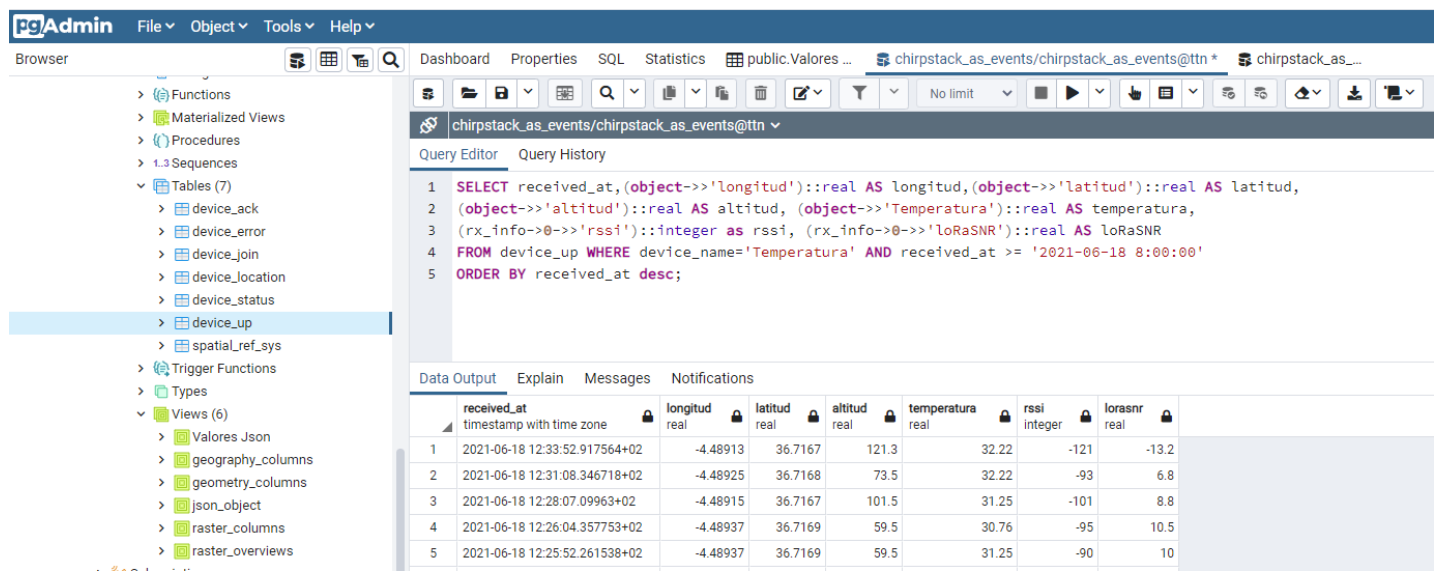

Figura 5: Gestor de base de datos PostgreSQL pgAdmin

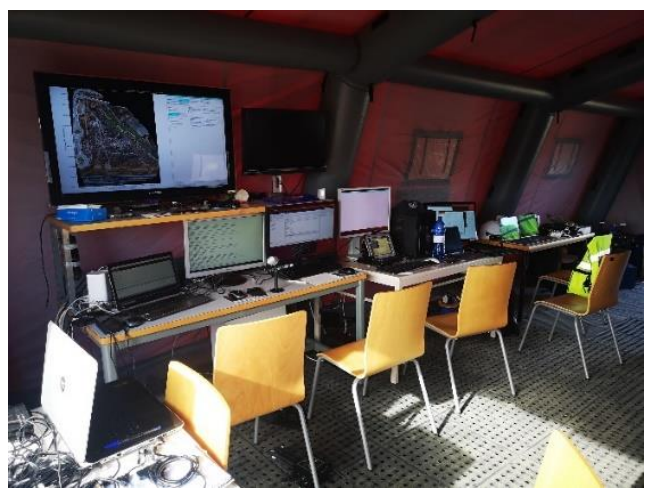

Figura 6: PCA del equipo de búsqueda y rescate

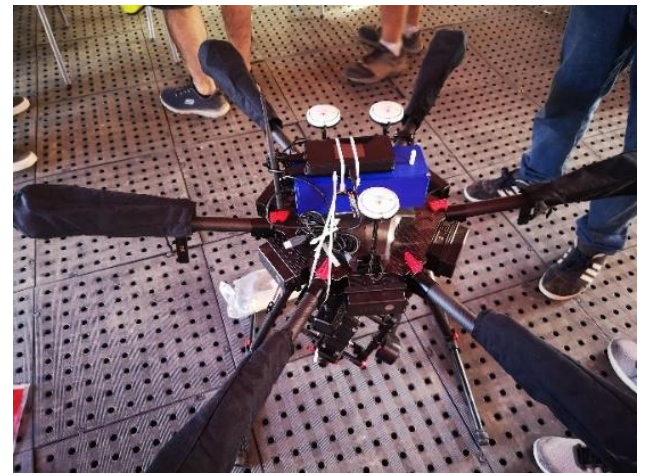

Figura 7: Nodo sensor montado en dron

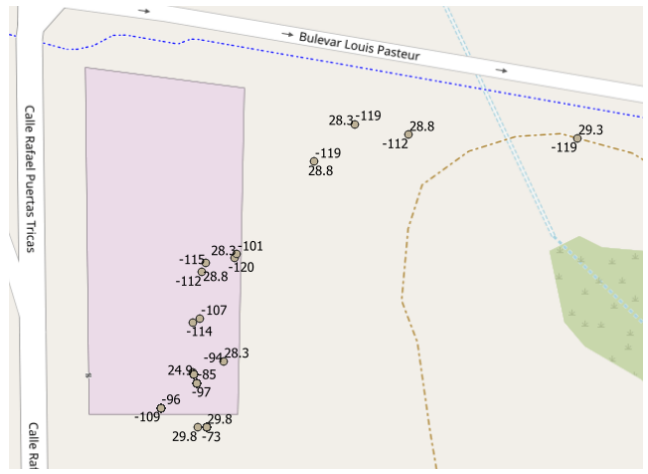

Figura 8: Visualización de información en QGIS

\section{CONCLUSIONES}

Las pruebas de campo permitieron demostrar que el sistema de comunicación de respaldo usando tecnología LoRa funciona correctamente.

La selección de la tecnología LoRa es adecuada para la aplicación ya que se consigue trasmitir datos con un largo alcance y, debido a que no requiere de servicios de terceros, implementar una red ad-hoc independiente de cualquier sistema pre-existente a la emergencia o catástrofe, como por ejemplo la infraestructura de las comunicaciones móviles.

En relación al diseño del nodo sensor, el peso y volumen reducido permitió acoplar una batería de larga duración (aunque también estaba disponible una toma de $18 \mathrm{~V}$ del dron), e instalarlo sin problemas tanto en un dron como en un robot terrestre.

Como líneas de trabajo futuro se plantea ampliar la evaluación a otro tipo de situaciones realistas, incluyendo, por ejemplo, oclusiones por escombros. Aunque la banda sub-GHz garantice una mejor penetrabilidad de señal que otras bandas, es interesante evaluar la atenuación en estos escenarios, e incluso en ambientes con gases, producto de incendios.

El código y la documentación del sistema presentado en este artículo está disponible en https://github.com/jjflozano/BackUpCommLoRa.

\section{Agradecimientos}

Este trabajo ha sido parcialmente financiado por los proyectosRTI2018-093421-B-I00, Ministerio de Ciencia, Innovación y Universidades, Programa Estatal de I+D+i 2018, y UMA18-FEDERJA-090, Programa operativo FEDER Andalucía 2014-2020, Convocatoria 2018, Junta de Andalucía. 


\section{English summary}

\section{BACKUP COMMUNICATION SYSTEM USING LORA TECHNOLOGY WITH HARDWARE AND OPEN SOFTWARE FOR EMERGENCY ROBOTICS APPLICATIONS}

\begin{abstract}
The communication systems between sensor nodes and the rescue teams present potential vulnerabilities in emergencies or disasters such as infrastructure failure or network saturation. Therefore, it is necessary to evaluate alternatives to guarantee the availability of the system and the data necessary for the rescue team's strategy. The requirements of the long-range and low-consumption network aim to evaluate the different technologies within LPWAN (Low Power, Wide Area Network). Among them, the LoRaWAN (Long Range, Wide Area Network) protocol offers operational flexibility that others do not have. On the hardware side, a selection of open components was made for the sensor nodes and the gateway. To achieve an Ad-hoc network, the ChirpStack project has been evaluated as a network and application server. Finally, the QGIS application software was integrated with the PostgreSQL database that stores the data in the same gateway host card. The system was tested at the XV International Conference of the University of Malaga on Security, Emergencies and Catastrophes. The system code and documentation presented in this article is available at https://github.com/jjflozano/BackUpCommLoRa.
\end{abstract}

Keywords: sensor node networks, mobile robots, LoRaWAN, SAR, backup communication system, adhoc network, Chirpstack.

\section{Referencias}

[1] R. R. Murphy, S. Tadokoro and A. Kleiner, "60. Disaster Robotics," in Handbook of Robotics, Berlin, Springer, 2016, pp. 1577-1604.

[2] F. Lara Hurtado, J. Lozano and R. Martín, "Una red de sensores inalámbrica basada en hardware y software abierto para colaboración con aplicaciones de robótica a misiones de búsqueda y rescate," in XL Jornadas de Automática, 2019.

[3] J. Fernandez-Lozano, A. Mandow, M. MartínGuzman, J. Martin-Ávila and J. Serón, "Integration of a Canine Agent in a Wireless
Sensor Network for Information Gathering in Search and Rescue Missions," in 2018 IEEE/RSJ International Conference on Intelligent Robots and Systems (IROS), Madrid, 2018.

[4] J. Bravo-Arrabal, J. J. Fernandez-Lozano, J. Serón, J. A. Gómez-Ruiz and A. García-Cerezo, "Development and Implementation of a Hybrid Wireless Sensor Network of Low Power and Long Range for Urban Environments," Sensors, vol. 21, no. 567, p. 27, 2021.

[5] R. Quinnell, "Low power wide-area networking alternatives," EDN Network, p. 14, 2015.

[6] Y. Song, J. Lin, M. Tang and S. Dong, "An Internet of Energy Things Based on Wireless LPWAN," Engineering, no. 3, p. 7, 2017.

[7] Ministerio de Energía, Turismo y Agenda Digital, BOE-A-2017-12318, Madrid: Gobierno Español, 2020.

[8] U. Raza, P. Kulkarni and M. Sooriyabandara, "Low Power Wide Area Networks: An Overview," IEEE, UK, 2016.

[9] W. Ayoub, F. Nouvel, A. Ellatif Samhat, J.-C. Prévotet and M. Mroue, "Overview and Measurement of Mobility in DASH7," 25th International Conference on Telecommunications (ICT), St. Malo, 2018.

[10] LoRa Alliance, "LoRaWAN What is it? A technical overview of LoRa and LoRaWAN," LoRa Alliance, San Ramon, CA, 2015.

[11] A. Augustin, J. Yi, T. Clausen and W. M. Townsley, "A Study of LoRa: Long Range \& Low Power Networks for the Internet of Things," Sensors, p. 18, 9 Septiembre 2016.

[12] G. Strazdins, A. Elsts, K. Nesenbergs and L. Selavo, "Wireless Sensor Network Operating System Design Rules Based on Real-World Deployment Survey," Journal of Sensor and Actuator Networks, pp. 509-556, 2013.

[13] A. Kifouche, G. Baudoin, R. Hamouche and R. Kocik, "Generic Sensor Network for Building Monitoring: Design, Issues, and Methodology," IEEE Conference on Wireless Sensors, pp. 3439, 2017.

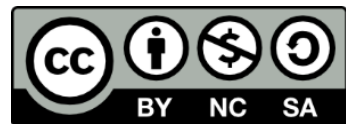

(C) 2021 by the authors. Submitted for possible open access publication under the terms and conditions of the Creative Commons Attribution CC BY-NC-SA 4.0 license (https://creativecommons.org/licenses/byncsa/4.0/deed.es 